\title{
Tide-topography interaction?
}

Leo R.M. Maas ${ }^{1}$ and Uwe Harlander ${ }^{2}$

1) NIOZ Royal Netherlands Institute for Sea Research, P.O. Box 59, 1790 AB, Texel, the Netherlands, maas@nioz.nl

2) Department of Aerodynamics and Fluid Mechanics, Brandenburg University of Technology (BTU) Cottbus, Germany, haruwe@tu-cottbus.de

\begin{abstract}
An analytical method is reviewed that enables the construction of two-dimensional topographies for which tidal conversion is absent. The method employs the analogue of conformal mapping to solve the tide-generation problem exactly. In this problem, a spatial hyperbolic equation governs the streamfunction field that reaches constant values at bottom and surface, and that is subject to a radiation condition far away from any topographic features present. This problem in principle governs both the trapped, nonhydrostatic (depth-dependent) barotropic, as well as propagating, internal wave response. But, employing the mapping, only the former, trapped field is obtained. For a particular class of localized ridges, we here verify this by constructing the same nonhydrostatic barotropic field in an entirely independent manner.
\end{abstract}

\section{Introduction}

One of us wrote a PhD thesis on 'Tide-topography interaction' (Maas, 1987). Now, twenty four years later, the only thing we have been able to add is a question mark ... Why? Surely, in a uniformly-stratified sea the flow of the surface tide over a ridge, as in Fig. 1a, will generate internal tides, right? Wrong! At least, for the particular bottom shape shown here. This is evident from the trapped nature of the baroclinic response,

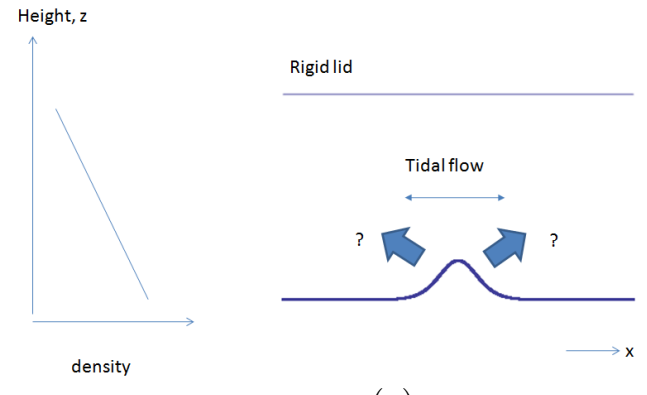

(a)

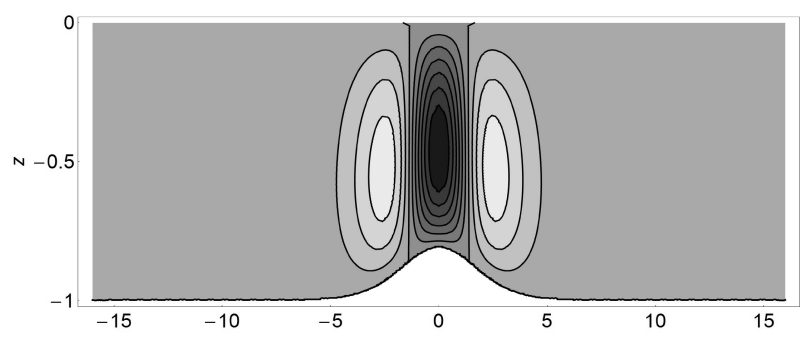

(b) ${ }^{x}$

Figure 1: (a) Tidal motion generates internal tides? (b) Baroclinic streamfunction response: no radiation of internal tides! Ridge height is determined by $\delta=1 / 2$.

shown in Fig. 1, that is nicely vanishing at top and bottom. It turns out that, in this case, there is not much interaction taking place after all. The complete absence of tidal conversion should not come as too big of a surprise as it was obtained before, e.g. over some piecewise, ridge-like topographies in Pétrélis et al. (2006). There it was attributed to annihilation of the internal tide generated on one side of the ridge, by that propagating in the same direction, but generated on the other side, see red arrows in Fig. 2. The part of the wave generated at the latter location, that moves in the opposite direction (inclined 
blue arrow), leads to a trapped response over the ridge. This explanation requires that reflecting rays (black lines) cover the ridge in an exact, even number of steps. However, it is not clear how this should apply to the continuous bottom profile, shown in Fig. 1b, that has 'neither beginning nor end'. So, how is this shape of bottom constructed in the first place, and can we see whether the same explanation, based on annihilation, is in order?

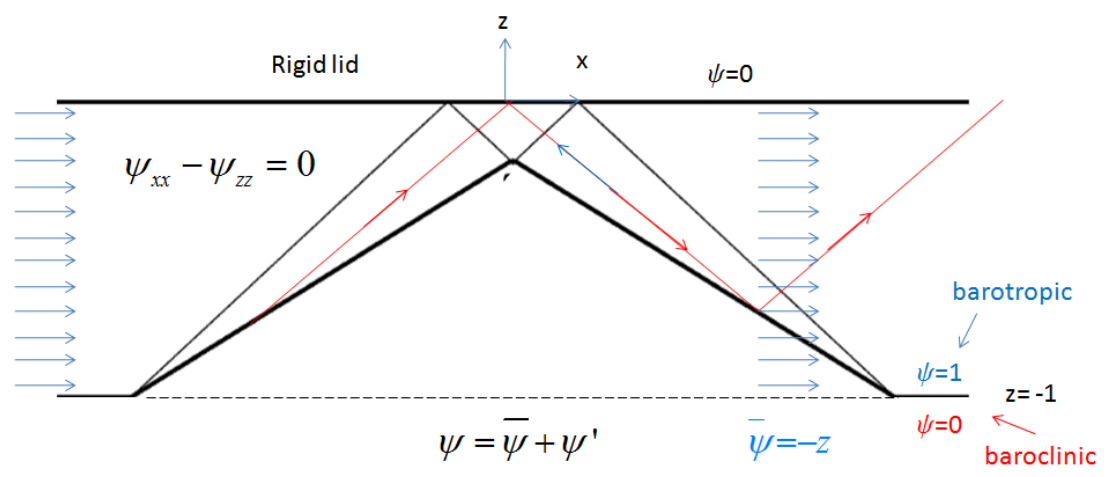

Figure 2: Sketch of characteristic (ray) structure over triangular ridge and formulation of tide-topography interaction problem (see text).

\section{Conversionless bottom shapes}

\subsection{Tide-topography interaction model}

The internal-tide generation model (Fig. 2), employs a rigid-lid surface, a uniform stratification and a back-and-forth barotropic tidal oscillation, $\propto \exp (-i t)$. Upon scaling, and stretching of the horizontal direction, a two-dimensional formulation allows the introduction of a streamfunction, $\psi(x, z) \exp (-i t)$, whose spatial part needs to satisfy the spatial wave equation

$$
\psi_{x x}-\psi_{z z}=0,
$$

where subscripts denote derivatives. Because we will here look at the response of a ridge, in our nondimensional formulation we use ocean depth, far away from the ridge, as length scale. The ratio of tidal frequency $\omega$ over (constant) buoyancy frequency $N$ implies an inclination along which internal wave energy propagates. By stretching the horizontal relative to the vertical, this inclination attains an angle of $45^{\circ}$, which is the direction of the characteristics of Eq. (1), $x \pm z=$ constant. Nondimensional parameters specify ridge properties, such as its width $L$ and height $\delta$. Upon rescaling, perturbation pressure and streamfunction appear as two 'hyperbolically conjugate' functions (Maas, 2011).

The hyperbolic equation (1) is to be solved subject to the conditions $\psi=0$, at the rigid lid $z=0$, and $\psi=1$, at the bottom $z=H(x)<0$. The latter describes the constant transport associated with the back-and-forth motion of the oscillatory, barotropic surface tide. In addition, a radiation condition at infinity, $x \rightarrow \pm \infty$, needs to be satisfied, stating that incoming internal tides are absent. However, for a large class of topographies, there is no internal tide generated at all. Only a trapped baroclinic response is obtained (Maas, 2011). Here, baroclinic and barotropic refer to the $z$-dependent and $z$-independent parts of the corresponding horizontal velocity field respectively. It turns out this terminology is not entirely adequate. The trapped, $z$-dependent horizontal velocity field is better interpreted as the nonhydrostatic barotropic response (Garrett and Gerkema, 2007). It happens to be 
the only part obtained with the method discussed next, in which the genuine baroclinic response, consisting in outward propagating internal waves, is absent.

\subsection{Mapping the wave equation}

A solution of the tide-topography interaction problem, as formulated in the previous subsection, is found by mapping the Cartesian $(x, z)$ coordinate frame onto a transformed plane by choosing new coordinates, $(\xi, \zeta)$, to obey hyperbolic Cauchy-Riemann equations,

$$
\left\{\begin{array}{l}
\xi_{x}=\zeta_{z} \\
\xi_{z}=\zeta_{x}
\end{array}\right.
$$

Using this transformation, the original problem is mapped onto the very same problem in the new coordinate frame,

$$
\psi_{\xi \xi}-\psi_{\zeta \zeta}=0
$$

provided a multiplicative factor, the Jacobian of transformation, $J \equiv J(\xi, \zeta)=\xi_{x} \zeta_{z}-\xi_{z} \zeta_{x}$, is nonzero. Since both $\xi$ and $\zeta$ again obey wave equation, (1), a general solution of (2), mapping the rigid-lid surface, $z=0$, onto a rigid-lid surface in the transformed plane, $\zeta=0$, reads

$$
\left\{\begin{array}{l}
\xi=f(x+z)+f(x-z) \\
\zeta=f(x+z)-f(x-z) .
\end{array}\right.
$$

Here, $f(q)$ is arbitrary provided it has non-vanishing first and second-derivatives. Using (4), Jacobian $J=4 f^{\prime}(x+z) f^{\prime}(x-z)$ is nonzero when $f^{\prime}(q) \neq 0$, where a prime denotes a derivative to its argument. This condition is satisfied for subcritical bottoms (Maas, 2011), whose slope is everywhere less than that of the characteristics.

In the transformed plane, the boundary condition at bottom $\zeta=h(\xi)<0$, still reads, $\psi=1$. We here choose the transformed ocean to have depth, $h=$ constant $<0$, a constant that, in view of the scaling, is simply $h=-1$. In the original, Cartesian frame, the bottom shape is given implicitly by $\zeta=h$, or $f(x+z)-f(x-z)=-1$, which is sometimes made explicit, as $z=H(x)$, or inversely as $x=\bar{H}(z)$.

There is no tidal conversion in the transformed plane since (3) can be solved trivially using separation of variables and all we get in solving the forced problem is the trivial barotropic solution, $\psi=\zeta / h$. But, in the original frame, $\psi$ obtains a nontrivial, albeit still nonpropagating shape, that needs to be interpreted as barotropic.

\section{Example: ridge of infinite support}

Choosing, for example, $f(q)=[q+\delta \tanh (q / 2)] / 2$, using (4), the transformed coordinates read

$$
\left\{\begin{array}{l}
\xi(x, z)=x+\delta \frac{\sinh x}{\cosh z+\cosh x} \\
\zeta(x, z)=z+\delta \frac{\sinh z}{\cosh z+\cosh x} .
\end{array}\right.
$$

Eq. (4b) implies that bottom, $z=H(x)$, is determined by solving $\zeta=-1$ for $z$ as a function of $x$. Explicitly, this gives the bottom shape (inversely) as $x=\bar{H}(z)$, where

$$
\bar{H}(z)= \pm \operatorname{arccosh}\left(-\frac{\delta}{1+z} \sinh z-\cosh z\right) .
$$

For $|x| \rightarrow \infty$, ocean depth $H \rightarrow-1$. At $x=0$, its depth $H_{0}$ reaches a minimum, determined by $H_{0}+\delta \tanh H_{0}+1=0$. Ridge height, $H_{0}+1$, is controlled by $\delta$. 
Over ridge (6), the tide-topography 'interaction' problem is thus, using (5b), solved by $\psi=-\zeta(x, z)$. The streamlines follow the bottom, and blend in smoothly with the straight, rigid surface. It is only by subtracting $\tilde{\psi} \equiv z / H(x)$, which has a verticallyuniform horizontal flow field, $u=-\tilde{\psi}_{z}$, that the strictly trapped nature of the 'baroclinic' response over this ridge, $\Psi^{\prime}=\psi-\tilde{\psi}$, becomes evident, see Fig. 1b as this separation implies $\Psi^{\prime}=0$ at the boundaries.

The question of interest is whether the absence of tidal conversion over this ridge can be understood as annihilation, as for the simple triangular ridge profile considered in Pétrélis et al. (2006). It is difficult to say so, as the ridge is of infinite support. From this perspective, another example, that considers a ridge of finite support, is perhaps more fruitful.

\section{Example: ridge of finite support}

A ridge of finite support of dimensionless width, $L$, and height related to $\delta$, again lacking tidal conversion, is obtained taking (Maas, 2011)

$$
f(q)= \begin{cases}\frac{1}{2(1-\delta)}\left(q+\frac{\delta L}{\pi} \sin \left(\pi \frac{q}{L}\right)\right), & |q|<L \\ \frac{1}{2}\left(q \pm \frac{\delta L}{1-\delta}\right), & \pm q \geq L .\end{cases}
$$

The ridge itself is slightly more complicated to define, owing to the piecewise definition of $f(q)$ and the fact that the bottom is defined through a difference equation, involving two such functions (details will be presented elsewhere). Figure 3a, shows that its 'baroclinic' field has a triple-cell structure very similar to that in Fig. 1b, albeit now confined exactly to the finite-sized ridge. To see whether the apparent annihilation beyond the ridge

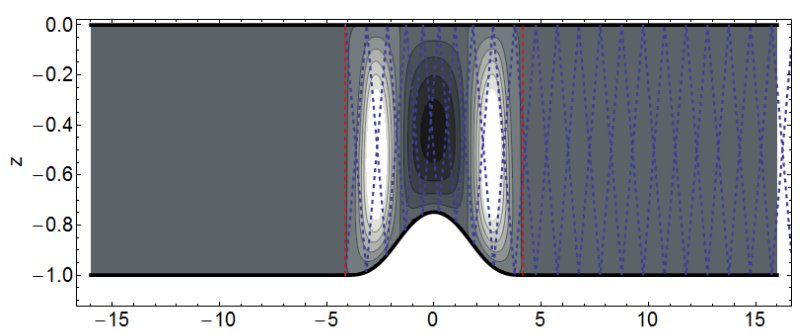

(a)

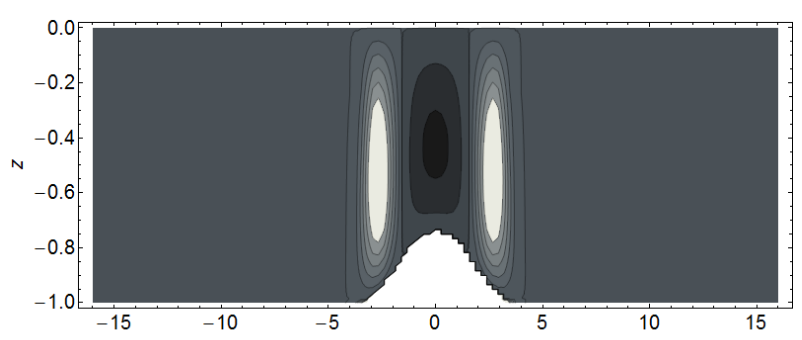

(b)

Figure 3: (a) Sketch of baroclinic streamfunction field, $\Psi^{\prime}$ and characteristic half-webs (blue dashed lines) over ridge of finite support having $\delta=0.15$ and $L=\pi$. Edges of the ridge, at $x= \pm X$, are indicated by red vertical dashed lines. (b) Baroclinic streamfunction field computed using alternative method over ridge of finite support having $\delta=0.15$ and $L=\pi$.

region, $|x|>X \equiv 1+L$, is due to a precise match of the characteristics, as for the triangular ridge, let us plot some characteristic (half) webs. Here, a characteristic web is understood as a certain characteristic and all of it predecessors and successors upon subsequent reflections from surface and bottom. Surprisingly, in general there is no such match for the ridge of finite support. Fig. 3a shows that, while starting half-webs at the left 'edge' of the ridge, indicated by a red vertical dashed line, both at the surface, $(-X, 0)$, and bottom, $(-X,-1)$, these characteristic half-webs do not incorporate $(X, 0)$ or $(X,-1)$, at the right edge, again indicated by a red vertical dashed line. This makes the observed apparent annihilation enigmatic. In the next section we discuss an alternative to the map method of section 2, which shows in what sense annihilation yet occurs and what particular circumstance seems to give rise to it. 


\section{Alternative solution method}

As an alternative to the map method we now use that for free internal waves, characteristic webs possess a web invariant, the partial pressure (Maas and Lam, 1995). This terminology derives from the fact that the pressure due to internal waves in a certain point is simply the sum of the partial pressures on its two unique intersecting characteristics. The corresponding streamfunction, being hyperbolically conjugate to the pressure, is the difference between these partial pressures. A point on the boundary, however, has two characteristics belonging to the same web. Their difference is therefore zero at the boundary, guaranteeing that for free waves the streamfunction vanishes along the boundary.

However, this changes when the internal wave field is forced (Maas and Lam, 1995). Consider forcing over some segment of the boundary where the streamfunction is nonzero. As a consequence, the partial pressures on the two half-webs, emanating from any point of that segment, can no longer be identical. When the two are real, the baroclinic wave field is standing: a blinking oscillatory type of response to the boundary forcing. This kind of forcing provides an alternative way to obtain solutions for the tide-topography interaction over a ridge of finite support. To see this, consider the decomposition of the streamfunction field indicated in Fig. 2. There, streamfunction field $\psi=\bar{\psi}+\psi^{\prime}$ is written as, $\bar{\psi}=-z$, a solution for the tide-topography interaction problem over a comparison topography, viz. the channel of uniform depth, plus a residual $\psi^{\prime}$. The residual $\psi^{\prime}$ is still determined by the homogeneous wave equation (1), but its boundary forcing is now localized. It is restricted to the ridge region, $|x| \leq X$, where

$$
\psi^{\prime}=\psi_{b} \equiv 1+H(x), \quad \text { at } z=H(x)
$$

Thus it is immediately in the framework just discussed. To proceed, consider that the general solution of the spatial wave equation reads $\psi^{\prime}=\phi(x+z)-\gamma(x-z)$, in terms of two arbitrary functions $\phi$ and $\gamma$, both representing the partial pressure $P$ on their respective characteristics. Suppose we aim to determine streamfunction $\psi^{\prime}$ and perturbation pressure $p^{\prime}$ in one particular point inside the fluid domain, $(\hat{x}, \hat{z})$ say. The invariants on the four half-webs through this point, that reflect from surface and bottom, will determine these fields as follows. Each half-web will have reflections from the flat parts of surface and bottom, where $\psi^{\prime}=0$, hence no forcing of the wave field. The partial pressure is set only at points along the ridge. Different such points on a particular half-web will provide different additive contributions to the value obtained at $(\hat{x}, \hat{z})$ :

$$
\psi^{\prime}(\hat{x}, \hat{z})=\sum_{n=1}^{N^{+-}} P_{n}^{+-}+\sum_{n=1}^{N^{-+}} P_{n}^{-+}-\sum_{n=1}^{N^{++}} P_{n}^{++}-\sum_{n=1}^{N^{--}} P_{n}^{--},
$$

with a similar expression for $-p^{\prime}$, except that all four terms are addes. Here $N^{ \pm \pm}$gives the number of boundary points along the half-webs that approach $(\hat{x}, \hat{z})$ from quadrants labeled ++ (upper right), +- (lower right), -- (lower left) and -+ (upper left). $P_{n}^{ \pm \pm}$ gives the contribution by the $n$-th reflection from the ridge along that half-web.

This contribution is given as follows. Since the difference in partial pressures determines the streamfunction, according to (8) this implies that at a boundary forcing point we have to take partial pressures $\phi=-\gamma=\frac{1}{2} \psi_{b}$. These functions are therefore real, which has the consequence that the solution that we thus construct is also real.

To construct the perturbation streamfunction field in the fluid domain covering the range $-16<x<16$ and $-1<z<0$, we use a grid of $120 \times 60(\hat{x}, \hat{z})$-points in horizontal 
and vertical directions respectively. For comparison with the result obtained with the map, section 2 , we first add $\bar{\psi}=-z$ to (9) and obtain streamfunction $\psi$, and subsequently subtract $\tilde{\psi}$, yielding $\Psi^{\prime}$. The field in Fig. $3 \mathrm{~b}$ is identical to the one constructed by use of the map (Fig. 3a) and thus validates the construction of this real-valued streamfunction field by superposition of locally forced waves.

\subsection{Characteristic webs}

Inspection of the characteristic half-webs, such as in Fig. 3a, shows that when our evaluation point $(\hat{x}, \hat{z})$ lies to the right of the ridge, $\hat{x}>X$, only terms along the characteristics coming from the left (indices - \pm ) contribute to its streamfunction. For the present choice of ridge depth and length, there are $N^{- \pm}=4$ or 5 forcing points along the two particular half-webs. Thus, eight to ten boundary points in total contribute to the pressure at $(\hat{x}, \hat{z})$. Remarkably, the combined partial pressures cancel exactly when evaluating streamfunction (9), not just in this single point but for any point having $\hat{x}>X$ (or $\hat{x}<-X$ ). The annihilation implies that, upon passing the ridge, the additive partial pressures on all half-webs are identical. This is not obvious at all. There is no clear symmetry involved. It is reminiscent of a similar result, described in Maas and Lam (1995), where integral properties of webs also appeared to be identical, albeit in that case over some part of a parameter plane. A clue to the reason for this surprising kind of annihilation may perhaps

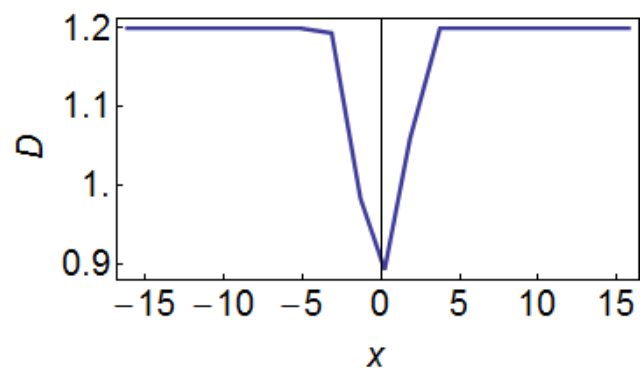

(a)

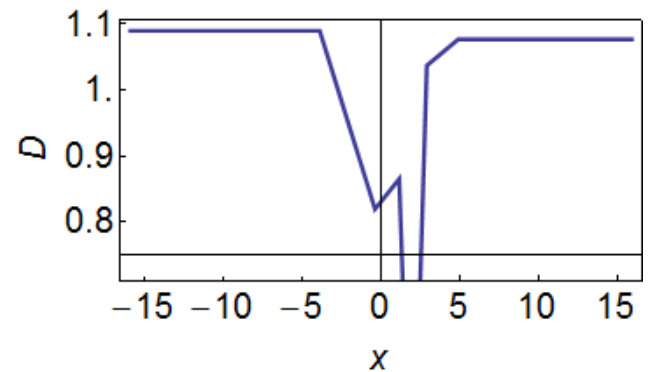

(b)

Figure 4: Along-surface distance $D$ between two characteristics plotted at average positions as a function of their horizontal position $x$ over the ridge of finite support given by (a) $H(x)$, (discussed elsewhere) having $\delta=0.15$ and $L=\pi$, and (b) $H_{c}(x)$, Eq. (10), having $\epsilon=0.122$ and $L=\pi$.

be related to a peculiar property of the characteristic webs. When two webs are followed from left to right, while passing the ridge, their distance $D$ (measured between reflection points at the surface) decreases. However, after passing the ridge, the characteristics recover their original distance; see Fig. 4a. This property is shared by any two characteristics. In other words, topographies lacking tidal conversion also lack net focusing of characteristics. The absence of focusing is a feature having profound implications in enclosed fluid domains, where it led to the anomalous appearance of 'global resonances' (Maas and Lam, 1995) and appeared to play a key role in the absence of scattering of an internal wave mode incident on a 'rough' piece of topography as well (Bühler and Holmes-Cerfon, 2011). Here, we find it may also explain why tidal conversion may be absent for the class of topographies that can be obtained by mapping the spatial wave equation in an irregularly shaped ocean onto a constant depth ocean. 


\subsection{Cosine ridge}

To check this, let us look at another topography of finite support, namely an isolated cosine-shaped ridge, that smoothly blends in with a flat ocean (Sandstrom, 1976),

$$
H_{c}(x)= \begin{cases}-1+\epsilon\left(\cos \frac{\pi x}{X}+1\right), & |x|<X \\ -1, & |x|>X\end{cases}
$$

By proper choice of ridge height $\epsilon$, this follows the ridge of finite support quite closely. For this cosine ridge, the streamfunction for $|x|>X$ is no longer annihilated. This may be related to the fact that characteristics no longer retain their original distance upon passing this cosine ridge, as visible in Fig. $4 \mathrm{~b}$ when comparing distances to the right and left. While the streamfunction is still amplified around the ridge, it produces persistent 'wavy' components over the flat ocean parts. Because we here describe the forcing as a real function, the latter provides an unphysical, standing instead of propagating wave, which is merely blinking in time. In order to satisfy the radiation condition some transfer of energy into outward-propagating internal wave modes is required, such as accomplished by other methods (Sandstrom, 1976; Llewellyn Smith and Young, 2002). While these methods are satisfactory in providing amplitudes of outward-propagating wave modes, they leave the essentially physical question, on how the pressure acquires a component that is in-phase with the streamfunction, unanswered. In future work we aim to address this issue, using our alternative method, exposed above. In this method, we aim to formulate a radiation condition not infinitely far away from but at the source. This holds the promise of being applicable to finite-sized fluid domains, or to domains that do not revert to constant depth, as well.

\section{Conclusion}

A simple back-and-forth barotropic tidal flow over a topographic irregularity in uniformlystratified, rigid-lid fluids, in general leads to both trapped barotropic, as well as propagating baroclinic waves. For this tide-topography interaction, a recently developed method constructs a particular class of topographies for which the problem is solved strictly by a barotropic flow (Maas, 2011). The transformation employed picks those topographies that can be mapped onto simpler ocean domains. Returning to the physical domain, this solution acquires a $z$-dependent horizontal flow, yet should still be considered as barotropic but having a nonhydrostatic contribution (Garrett and Gerkema, 2007). As this solution to the tide-topography 'interaction' problem does not generate any propagating internal tides, these topographies are conversionless.

Here, we checked this finding by computing the response over one such conversionless topography - a ridge of finite support - using an alternative method. In this new method, the solution is constructed in a field point by using the property that the wave-induced pressure in such a point is the sum of the partial pressures on the two characteristic webs crossing this point. The partial pressure of a characteristic web is invariant, except when forced at one of its boundary points, where it can suddenly jump by an additional 'kick'. Such forcing takes e.g. place when there is a flow through the boundary.

Solving the tide-topography interaction problem in a comparison ocean of constant depth yields a simple horizontal barotropic oscillatory motion (horizontal arrows in Fig. 2). This produces an apparent flow through the actual bottom, driving the response over the ridge and beyond. This apparent flow provides additional contributions to the partial pressure of a particular characteristic web, wherever the web reflects from the ridge. 
Adding these, the partial pressure on this characteristic web outside the ridge region is fixed. The streamfunction field in a point is obtained as the difference of the partial pressures of these two characteristic webs. For conversionless topographies, outside the ridge region, these two contributions annihilate one another completely. The sole response of the fluid is an oscillatory, $z$-dependent motion, directly over the ridge, corresponding exactly to the nonhydrostatic barotropic field obtained using the mapping. It is an adiabatic change of the flow field, lacking conversion into internal tides.

For a converting, cosine-ridge of finite support, however, the streamfunction, following the same method, does not vanish outside the ridge region. This is unacceptable (it violates the radiation condition), and requires adapting the method, which we leave for future work. The different result over the two topographies is perhaps related to the remarkable property that two characteristic webs, upon passing a conversionless ridge, recover their original distance, while they do not when passing a converting ridge. The absence of conversion is indeed due to annihilation, but in a more subtle way than previously anticipated. The requirement that a ridge is passed by an exact even number of bounces, appears to be too stringent. Since the boundary forcing is proportional to local bottom depth, Eq. (8), this requirement is replaced by a functional equation that is apparently satisfied by the particular bottom shapes for which tidal conversion is absent.

\section{Acknowledgements}

This work was completed while the first author was on a sabbatical leave at the University of Porto. Hospitality provided by José da Silva is gratefully acknowledged.

\section{References}

Bühler, O. and Holmes-Cerfon, M. (2011). Scattering of internal tides by random topography. Journal of Fluid Mechanics, in press.

Garrett, C. and Gerkema, T. (2007). On the Body-Force Term in Internal-Tide Generation. Journal of Physical Oceanography, 37:2172.

Llewellyn Smith, S. G. and Young, W. R. (2002). Conversion of the barotropic tide. Journal of Physical Oceanography, 32(2):1554-1566.

Maas, L. R. M. (1987). Tide-topography interaction in a stratified shelf sea. PhD thesis, Utrecht University.

Maas, L. R. M. (2011). Topographies lacking tidal conversion. J. Fluid Mech., in press.

Maas, L. R. M. and Lam, F. P. A. (1995). Geometric focusing of internal waves. Journal of Fluid Mechanics, 300:1-41.

Pétrélis, F., Llewellyn Smith, S., and Young, W. R. (2006). Tidal Conversion at a Submarine Ridge. Journal of Physical Oceanography, 36(6):1053-1071.

Sandstrom, H. (1976). On topographic generation and coupling of internal waves. Geophysical and Astrophysical Fluid Dynamics, 7:231-270. 